Pacific Journal of Mathematics

COLLECTIVELY COMPACT SETS AND THE ERGODIC 


\title{
COLLECTIVELY COMPACT SETS AND THE ERGODIC THEORY OF SEMI-GROUPS
}

\author{
M. V. Deshpande
}

Let $\{T(t): t \geqq 0\}$ be a uniformly bounded semi-group of linear operators on a Banach space $X$ such that 1 is an eigenvalue of each $T(t)$ and $T(a)$ is compact for some $a>0$. Then the ergodic limit $A(t)=\lim _{n \rightarrow \infty}(1 / n)\left\{T(t)+T^{2}(t)+\cdots+\right.$ $\left.T^{n}(t)\right\}$ exists for each $t$. In this paper it is proved that if each $T(t), t>0$, is compact and 1 is, in a certain sense, an isolated eigenvalue of all $T(t)$, then for $t>0$, the dimension of the null space of $T(t)-I$ is independent of $t$. Sufficient conditions are also obtained for the $\lim _{t \rightarrow \infty} T(t)$ to exist.

Suppose $X$ is a real or complex Banach space. Let $B$ denote the unit ball in $X$ and [X] the space of bounded linear operators on $X$ into $X$. A set $\mathscr{K} \subset[X]$ is said to be collectively compact if $\mathscr{K} B=\{K x: K \in \mathscr{K}, x \in B\}$ is relatively compact. Basic properties of such sets were obtained by Anselone and Palmer $[1,2]$. Some of their results will be applied to semi-groups in the following sections.

2. Ergodic family associated with a semi-group. Let $\{T(t): t \geqq 0\}$ be a uniformly bounded semi-group of linear operators on a $B$-space $X$ such that $T(a)$ is compact for some $a>0$ and 1 is an eigenvalue of each $T(t)$. Then [4, VIII. 8.4] yields that

$$
\begin{aligned}
\lim _{n \rightarrow \infty} & \cdot(1 / n)\left\{T(t)+T^{2}(t)+\cdots+T^{n}(t)\right\} \\
& =\lim _{n \rightarrow \infty} \cdot(1 / n)\{T(t)+\cdots+T(n t)\} \\
& =A(t)
\end{aligned}
$$

say, exists in the uniform operator topology and defines a projection operator satisfying $A(t)=T(t) A(t)=A(t) T(t)$. Also, $A(t)$ is the residue operator in the Laurent expansion of $(\lambda-T(t))^{-1}$ in the neighbourhood of 1 and is represented by the Dunford integral

$$
A(t)=(1 / 2 \pi i) \int_{C}(\lambda-T(t))^{-1} d \lambda
$$

where $C$ is a sufficiently small circle with centre at 1 and will, in general, depend upon $t$. For $t=0, T(0)=A(0)=I$ the identity operator. $\{A(t): t \geqq 0\}$ will be called the ergodic family associated with $\{T(t): t \geqq 0\}$.

When $T(a)$ is compact for some $a>0$, the family $\{T(t): t \geqq a\}$ is collectively compact and totally bounded in the uniform operator 
topology [3]. But nothing can be said about $T(t)$ for $t<a$. However, the following proposition shows that the situation is more satisfactory in respect of the family $\{A(t)\}$.

Proposition 2.1. Let $\{T(t): t \geqq 0\}$ be a uniformly bounded semigroup on a $B$-space $X$ such that 1 is an eigenvalue of each $T(t)$ and $T(a)$ is compact for some $a>0$. Then for each $\varepsilon>0$, the ergodic family $\{A(t): t \geqq \varepsilon\}$ is collectively compact.

Proof. Since $A(t)=T(t) A(t)$, by repeated application, we have $A(t)=T(n t) A(t)$ for all positive integers $n$. For $t>0 T(n t)$ is compact for sufficiently large $n$. Hence, $A(t)$ will also be compact. Now for $\varepsilon>0$, choose a positive integer $k$ so that $k \varepsilon \geqq a$. Then $k t \geqq a$ for $t \geqq \varepsilon$. Therefore, $\mathscr{K}=\{T(k t): t \geqq \varepsilon\}$ is collectively compact. Now, $\|T(t)\| \leqq M$ implies $\|A(t)\| \leqq M$. Hence, $\mathscr{C}=\{A(t): t \geqq \varepsilon\}$ is uniformly bounded. We can now conclude that

$$
\mathscr{K} \mathscr{L C}=\{T(k t) \circ A(s): t, s \geqq \varepsilon\}
$$

is collectively compact, because, by [1, Prop. 2.3], if $\mathscr{K}$ is collectively compact and $\mathscr{C}$ is uniformly bounded, then $\mathscr{K} \mathscr{C}$ is collectively compact. The result now follows from the fact that $\{A(t): t \geqq \varepsilon\}$ is a subset of $\mathscr{K} \mathscr{A}$.

COROLLARY 2.2. The family $\{A(t): t \geqq \varepsilon\}$ is totally bounded in the uniform operator topology.

Proof. The above arguments in respect of $\{T(t)\}$ also hold for the dual semi-group $\left\{T^{*}(t)\right\}$. Hence, $\{A(t): t \geqq \varepsilon\}$ and $\left\{A^{*}(t): t \geqq \varepsilon\right\}$ are both collectively compact. The desired conclusion follows from the fact that if $\mathscr{K}_{,} \mathscr{K}^{*}$ are both collectively compact, then $\mathscr{K}$ is totally bounded in the uniform operator topology [6, Thm. 3.1].

3. Spectral properties. In Section 2 strong continuity of $T(t)$ was not necessary. But, for the spectral properties to be discussed now, we shall require strong continuity. Let, as usual, $\sigma(T(t))$, $\tilde{\rho}(T(t))$ denote the spectrum and the extended resolvent set of $T(t)$ and $R(\lambda, T(t))$ the inverse $(\lambda-T(t))^{-1}$. The following proposition proved in [3] leads to the strong continuity of $A(t)$ which is crucial for the main result.

Proposition 3.1. Let $\{T(t): t \geqq 0\}$ be a uniformly bounded, strongly continuous semi-group on a B-space $X$ such that $T(t)$ is compact for each $t>0$. For $a>0$, let $\Omega$ be a neighbourhood of $\sigma(T(a))$, and $\Lambda=\tilde{\rho}(T(a))-\Omega$. Then 
(i) there exists a $\partial>0$ such that, for $|t-a|<\partial, \sigma(T(t)) \subset \Omega$ and $\Lambda \subset \tilde{\rho}(T(t))$.

(ii) As $t \rightarrow a,(\lambda-T(t))^{-1} x \rightarrow(\lambda-T(a))^{-1} x$ uniformly on $\Lambda$.

Proposition 3.2. Let $\{T(t): t \geqq 0\}$ be a uniformly bounded, strongly continuous semi-group on a B-space $X$ such that 1 is an eigenvalue of each $T(t)$ and $T(t)$ is compact for each $t>0$. Suppose further, that for each $a>0$, there exists a circle $C$ with centre at 1 in the complex plane and a real number $\partial$ such that, for $|t-a|<\partial$, $T(t)$ has no eigenvalue in $C$ except 1 . Then for $t>0, A(t)$ is strongly continuous in $t$.

Proof. We know that

$$
A(t) x=(1 / 2 \pi i) \int_{\Gamma}(\lambda-T(t))^{-1} x d \lambda
$$

where, in general, $\Gamma$ depends upon $t$. But, under the hypothesis on $C$, we may assume that

$$
A(t) x=(1 / 2 \pi i) \int_{C}(\lambda-T(t))^{-1} x d \lambda
$$

for all $t$ with $|t-a|<\delta$. Again, by proposition 3.1, $(\lambda-T(t))^{-1} x \rightarrow$ $(\lambda-T(a))^{-1} x$ uniformly on $C$ as $t \rightarrow a$. Hence, for any $a>0$, we have $A(t) x \rightarrow A(\alpha) x$ as $t \rightarrow a$. This completes the proof.

Let $R(T), N(T)$ denote the range and null space of an operator $T$ and $\operatorname{dim} R(T), \operatorname{dim} N(T)$ their dimensions. When $T_{n} \rightarrow T$ pointwise, $\operatorname{dim} R\left(T_{n}\right)>\operatorname{dim} R(T)$ eventually. But, when we are dealing with projections, the following result $[2,4.2]$ gives a more precise estimate.

THEOREM 3.3. Let $E$ and $E_{n}, n \geqq 1$, be projections in [X] such that $E_{n} \rightarrow E$ pointwise and $\left\{E_{n}-E\right\}$ is collectively compact. Then, eventually $\operatorname{dim} E_{n} X=\operatorname{dim} E X$.

THEOREM 3.4. Let $\{T(t)\}$ be the semi-group of Proposition 3.2. Then for each $a>0$, there exists $\partial>0$ such that $\operatorname{dim} N(T(t)-I)=$ $\operatorname{dim} N(T(a)-I)$ whenever $|t-a|<\partial$.

Proof. Observe first that $R(A(t))=N(T(t)-I)$ since $A(t)$ is the ergodic limit of $T(t)$. Suppose that there does not exist any $\partial$ with the required property. Then, by using Proposition 2.1, it is possible to construct a sequence of projections for which the Theorem 3.3 will not be true. 
THEOREM 3.5. If $\{T(t)\}$ is the semi-group of Proposition 3.4, then for $t>0, \operatorname{dim} N(T(t)-I)$ is independent of $t$.

Proof. By the last theorem the function $t \rightarrow \operatorname{dim} N(T(t)-I)$ is continuous and since it takes only positive integral values, it must be a constant function.

4. The nature of $T(t)$ at $t=\infty$. The first result of this section will be a lemma proved in [5] by using collective compactness. However, the proof given here throws some light on the nature of $T(t)$ at $t=\infty$.

Lemma 4.1. Let $T \in[X]$ be such that $\left\{T^{n}: n \geqq 1\right\}$ is uniformly bounded and $T^{k}$ is compact for some $k \geqq 1$. Then $\left\{T^{n}: n \geqq 1\right\}$ is totally bounded in the uniform operator topology.

Proof. The conditions on $T$ ensure that $\sigma(T)$ is contained in the closed unit dise with centre 0 in the complex plane and that there are only a finite number of simple poles of $R(\lambda, T)$, say $\lambda_{1}, \cdots, \lambda_{p}$ on the circumference of the disc [4, VIII. 8.1]. If $A_{1}, \cdots, A_{p}$ are the residue operators in the Laurent expansion of $R(\lambda, T)$ in the neighbourhoods of $\lambda_{1}, \cdots, \lambda_{p}$ respectively, then by using the standard technics of operational calculus, it can be easily proved that

$$
T^{n} x=(1 / 2 \pi i) \int_{C_{0}} \lambda^{n} R(\lambda, T) x d \lambda+\lambda_{1}^{n} A_{1}(x)+\cdots+\lambda_{p}^{n} A_{p}(x)
$$

where $C_{0}$ is a circle with centre at 0 and radius less than 1 . Since $|\lambda|<1$ on $C_{0}$, the first term on the right tends to zero uniformly on $C_{0}$ and on bounded sets of $X$. Again $\left|\lambda_{\kappa}\right|=1$ for $k=1, \cdots, p$. Hence $\left\{\lambda_{k}^{n}: n \geqq 1, k=1, \cdots, p\right\}$ is totally bounded. It is easy to extract a sequence $\left\{n_{i}\right\}$ such that $\lambda_{k}^{n_{i}}$ converges to $\mu_{k}$ say, for $k=1$, $\cdots, p$. It then follows that

$$
T^{n_{i}} x \longrightarrow \mu_{1} A_{1}(x)+\cdots+\mu_{p} A_{p}(x) .
$$

The convergence is obviously uniform on bounded sets of $X$.

Proposition 4.2. Let $\{T(t): t \geqq 0\}$ be a uniformly bounded semigroup such that $T(a)$ is compact for some $a>0$. For any $t>0$, let $A_{1}=A_{1}(t), \cdots, A_{p}=A_{p}(t)$ be the residue operators in the Laurent expansion of $R(\lambda, T(t))$ in the neighbourhoods of its poles $\lambda_{1}, \cdots, \lambda_{p}$ say, lying on the circumference of the unit disc. Then, there exists a sequence $n_{i}=n_{i}(t)$ such that, as $n_{i} \rightarrow \infty, T\left(n_{i} t\right) x$ converges to a linear combination of $A_{1} x, \cdots, A_{p} x$ for each $x$. 
Proof. Follows immediately when the previous lemma is applied to $T(t)$.

Proposition 4.3. Let $\{T(t): t \geqq 0\}$ be a uniformly bounded semigroup on a B-space $X$ such that $T(a)$ is compact for some $a>0$. Suppose 1 is the only eigenvalue of $T(t)$ on the unit circle in the complex plane for each $t>0$. Then $\lim _{n \rightarrow \infty} T(n t)=A$ exists in the uniform operator topology for each $t>0$ and $A$ is independent of $t$.

Proof. When the conditions of the proposition are satisfied equation (I) of this section applied to $T(t)$ gives

$$
T(n t) x=T^{n}(t) x=(1 / 2 \pi i) \int_{C_{0}} \lambda^{n}(\lambda-T(t))^{-1} x d \lambda+A(t) x
$$

where $A=A(t)$ is the residue operator in the Laurent expansion of $(\lambda-T(t))^{-1}$ around 1 and $C_{0}$ is a circle with centre at 0 and radius less than 1. Now $|\lambda|<1$ on $C_{0}$ and therefore $\lambda^{n} \rightarrow 0$ as $n \rightarrow \infty$. Hence taking limits in the above equation as $n \rightarrow \infty$ we get $\lim _{n \rightarrow \infty} T(n t) x=A(t) x$, the limit being uniform on bounded sets of $X$. Now, to prove that $A(t)=A$ is independent of $t$ note that $A(t) A(s)=$ $\lim _{n \rightarrow \infty} T(n t) T(n s)=\lim _{n \rightarrow \infty} T(n(s+t))=A(s+t)=A(s) A(t)$. Hence $\{A(t)\}$ is a semi-group. Again, for $t \neq s$, let $t<s$. Then by the semigroup property $R(A(t)) \supset R(A(s)$ ) where $R$ denotes the range. Also $A(t)$ is a projection. Therefore $A(t)=A^{n}(t)=A(n t)$ for each positive integer $n$. Choose $n$ so that $n t>s$. Then we have $R(A(t))=$ $R(A(n t)) \subset R(A(s))$. Hence $R(A(t))=R((A(s))$. Now, it is easy to prove that if $P, Q$ are projections such that $P Q=Q P$ and $R(P)=R(Q)$ then $P=Q$. Therefore we must have $A(t)=A(s)$. This completes the proof.

CoRollary 4.4. $N(T(t)-I)$ is independent of $t$ for $t>0$.

Proof. An application of corollary, Theorem 2 in [7, VIII, § 3] leads to $N(T(t)-I)=R(A(t))=R(A)$.

REMARK 4.5. In the proof of the Proposition $4.3 A(t)$ is proved to be independent of $t$ by using the fact that $A(t) A(s)=A(t+s)=$ $A(s) A(t)$ and that $A(t)$ is a projection. This means that the only semi-group of projections is the semi-group $T(t)=P$ a projection for all $t$.

In the next proposition it will be seen that the pointwise convergence of $T(n t)$ in $t$ proved in Proposition 4.3 can be strengthened so as to be locally uniform in $t$. This essential for the final ergodic theorem. 
Proposition 4.6. Let $\{T(t): t \geqq 0\}$ be a uniformly bounded, strongly continuous semi-group on a B-space $X$ such that $T(t)$ is compact for each $t>0$. Suppose there exists a circle in the complex plane with centre at 0 and radius less than 1 such that 1 is the only eigenvalue of each $T(t)(t>0)$ outside this circle. Then for each $t>0, \lim _{n \rightarrow \infty} T(n t)=A$ exists in the uniform operator topology, the convergence being locally uniform on $t$, i.e., for each $a>0$ there exists a positive $\partial$ depending upon a such that the convergence is uniform on the interval $|t-a|<\partial$.

Proof. The equation (II) of this section is now

$$
T(n t) x=(1 / 2 \pi i) \int_{C_{0}} \lambda^{n}(\lambda-T(t))^{-1} x d \lambda+A x
$$

where in general $C_{0}$ depends upon $t$. But, under the hypothesis made, $C_{0}$ may be assumed to be independent of $t$. Then by Proposition 3.1 the set $\left\{(\lambda-T(t))^{-1}: \lambda \in C_{0},|t-a|<\partial\right\}$ is uniformly bounded for some $\partial>0$, i.e., $\left\|(\lambda-T(t))^{-1}\right\|<M$ say, for all $\lambda \in C_{0}$ and $|t-a|<\partial$. Hence by equation (III) we have

$$
\begin{aligned}
\|T(n t) x-A x\| & \leqslant|(1 / 2 \pi i)| \int_{C_{0}}\left\|\lambda^{n}(\lambda-T(t))^{-1} x\right\||d \lambda| \\
& <(M / 2 \pi) \int_{C_{0}}|\lambda|^{n}|d \lambda| \\
& \longrightarrow 0 \text { as } n \longrightarrow \text { since }|\lambda|<1 \text { on } C_{0} .
\end{aligned}
$$

It is obvious that the convergence is of the required type.

Lemma 4.7. Suppose $\{T(t): t \geqq 0\}$ is a family of bounded linear operators on a B-space $X$ such that $\lim _{n \rightarrow \infty} T(n t)=A$ exists in the uniform operator topology where $A$ is independent of $t$ and the convergence is uniform on some open interval. Then $\lim _{t \rightarrow \infty} T(t)=A$ in the uniform operator topology.

Proof. We may assume that $T(n t) \rightarrow A$ uniformly on $|t-a|<\partial$. Then for $\varepsilon>0$, there exists $\partial>0$ and a positive integer $N$ such that $\|T(n t)-A\|<\varepsilon$ for all $n \geqq N$ and all $t$ in $|t-a|<\partial$ or equivalently $\|T(t)-A\|<\varepsilon$ for $n \geqq N$ and $|t-n a|<n \partial$. Let $I_{n}$ be the interval $(n a-n \partial, n a+n \partial)$. Then $I_{n}$ has a nonempty intersection with $I_{n+1}$ if and only if $n a+n \partial>(n+1) \alpha-(n+1) \partial$ or equivalently $(2 n+1) \partial>a$. Choose $M$ so that $n \geqq M$ implies $n \geqq N$ and $(2 n+1) \partial>a$. Then, for each $n \geqq M, I_{n}$ and $I_{n+1}$ have a nonempty intersection. Hence, any $t>M a-M \partial$ falls in some $I_{n}$ with $n \geqq M$. Therefore we must have $\|T(t)-A\|<\varepsilon$ for $t>M a-M \partial$. This completes the proof. 
In the light of this lemma, the Proposition 4.6 and the Corollary 4.4, the following ergodic theorem now becomes obvious.

THEOREM 4.8. If $\{T(t): t \geqq 0\}$ is the semi-group of Proposition 4.6 then $\lim _{t \rightarrow \infty} T(t)$ exists in the uniform operator topology, and $N(T(t)-I)$ is constant for $t>0$.

REMARK. In $\S \S 3$ and 4 the continuity of $R(\lambda, T(t))$ in $t$ plays a crucial role. If it is assumed that $T(t)$ is compact for each $t>0$, then the strong continuity of $T(t)$ in $t$ implies uniform continuity and this in turn implies the uniform continuity of $R(\lambda, T(t))$ for $\lambda \in \rho(T(t))$. But if instead of assuming the compactness of $T(t)$ for each $t>0$, it is assumed that for each $t>0$ there exists $\partial>0$ such that $\{T(t)-T(a):|t-a|<\partial\}$ is collectively compact then the strong continuity of $T(t)$ would not imply uniform continuity but the theory of collectively compact operators can be invoked to ensure the strong continuity of $R(\lambda, T(t))$.

\section{REFERENCES}

1. P. M. Anselone and T. W. Palmer, Collectively compact sets of linear operators, Pacific J. Math., 25 (1968) 417-23.

2. - Spectral analysis of collectively compact, strongly convergent operator sequences, Pacific J. Math., 25 (1968), 423-31.

3. M. V. Deshpande and N. E., Joshi, Collectively compact sets and semi-groups of linear operators, Pacific J. Maths., (To appear).

4. N. Dunford and J. T. Schwartz, Linear Operators Part-I, New York, Interscience, 1958.

5. J. A. Higgins, Collectively Compact Sets of Linear Operators, Ph.D. Dissertation, New Mexico State University, 1971.

6. T. W. Palmer, Totally bounded sets of pre-compact operators, Proc. Amer., Math. Soc., 22 (1969), 101-106.

7. K. Yosida Functional Analysis, Springer-Verlag, (1968).

Received February 27, 1974.

NagPur University CAMPUS,

NAGPUR, MaHARASHT INDia 



\title{
PACIFIC JOURNAL OF MATHEMATICS
}

\section{EDITORS}

RichaRd ARENS (Managing Editor)

University of California

Los Angeles, California 90024

\section{R. A. Beaumont}

University of Washington

Seattle, Washington 98105
J. DUGUNDJI

Department of Mathematics University of Southern California Los Angeles, California 90007

D. Gilbarg and J. Milgram

Stanford University

Stanford, California 94305

\section{ASSOCIATE EDITORS}
E. F. BECKENBACH
B. H. NeUmanN
F. WOLF
K. YoshIDA

\section{SUPPORTING INSTITUTIONS}

\author{
UNIVERSITY OF BRITISH COLUMBIA \\ CALIFORNIA INSTITUTE OF TECHNOLOGY \\ UNIVERSITY OF CALIFORNIA \\ MONTANA STATE UNIVERSITY \\ UNIVERSITY OF NEVADA \\ NEW MEXICO STATE UNIVERSITY \\ OREGON STATE UNIVERSITY \\ UNIVERSITY OF OREGON \\ OSAKA UNIVERSITY
}

\author{
UNIVERSITY OF SOUTHERN CALIFORNIA \\ STANFORD UNIVERSITY \\ UNIVERSITY OF TOKYO \\ UNIVERSITY OF UTAH \\ WASHINGTON STATE UNIVERSITY \\ UNIVERSITY OF WASHINGTON \\ AMERICAN MATHEMATICAL SOCIETY
}

The Supporting Institutions listed above contribute to the cost of publication of this Journal, but they are not owners or publishers and have no responsibility for its content or policies.

Mathematical papers intended for publication in the Pacific Journal of Mathematics should be in typed form or offset-reproduced, (not dittoed), double spaced with large margins. Underline Greek letters in red, German in green, and script in blue. The first paragraph or two must be capable of being used separately as a synopsis of the entire paper. Items of the bibliography should not be cited there unless absolutely necessary, in which case they must be identified by author and Journal, rather than by item number. Manuscripts, in triplicate, may be sent to any one of the editors. Please classify according to the scheme of Math. Reviews, Index to Vol. 39. All other communications should be addressed to the managing editor, or Elaine Barth, University of California, Los Angeles, California, 90024.

The Pacific Journal of Mathematics expects the author's institution to pay page charges, and reserves the right to delay publication for nonpayment of charges in case of financial emergency.

100 reprints are provided free for each article, only if page charges have been substantially paid. Additional copies may be obtained at cost in multiples of 50 .

The Pacific Journal of Mathematics is issued monthly as of January 1966. Regular subscription rate: $\$ 72.00$ a year (6 Vols., 12 issues). Special rate: $\$ 36.00$ a year to individual members of supporting institutions.

Subscriptions, orders for back numbers, and changes of address should be sent to Pacific Journal of Mathematics, 103 Highland Boulevard, Berkeley, California, 94708.

PUBLISHED BY PACIFIC JOURNAL OF MATHEMATICS, A NON-PROFIT CORPORATION

Printed at Kokusai Bunken Insatsusha (International Academic Printing Co., Ltd.), 8-8, 3-chome, Takadanobaba, Shinjuku-ku, Tokyo 160, Japan.

Copyright (C) 1975 by Pacific Journal of Mathematics Manufactured and first issued in Japan 


\section{Pacific Journal of Mathematics}

\section{Vol. 59, No. $2 \quad$ June, 1975}

Aharon Atzmon, A moment problem for positive measures on the unit disc ........

Peter W. Bates and Grant Bernard Gustafson, Green's function inequalities for

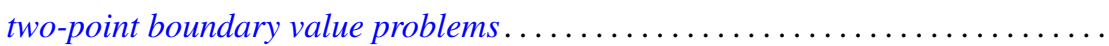

Howard Edwin Bell, Infinite subrings of infinite rings and near-rings ...........

Grahame Bennett, Victor Wayne Goodman and Charles Michael Newman, Norms of

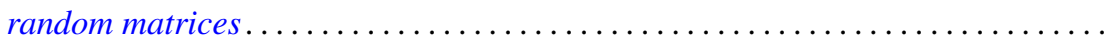

Beverly L. Brechner, Almost periodic homeomorphisms of $E^{2}$ are periodic.........

Beverly L. Brechner and R. Daniel Mauldin, Homeomorphisms of the plane ........

Jia-Arng Chao, Lusin area functions on local fields ......................

Frank Rimi DeMeyer, The Brauer group of polynomial rings ...............

M. V. Deshpande, Collectively compact sets and the ergodic theory of

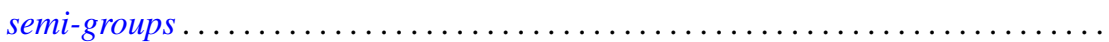

Raymond Frank Dickman and Jack Ray Porter, $\theta$-closed subsets of Hausdorff

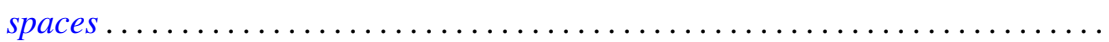

Charles P. Downey, Classification of singular integrals over a local field .......... 407

Daniel Reuven Farkas, Miscellany on Bieberbach group algebras . . . . . . . . . . . .

Peter A. Fowler, Infimum and domination principles in vector lattices . . . . . . . . . .

Barry J. Gardner, Some aspects of T-nilpotence. II: Lifting properties over

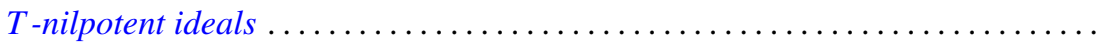

Gary Fred Gruenhage and Phillip Lee Zenor, Metrization of spaces with countable

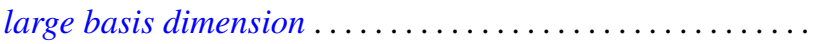

J. L. Hickman, Reducing series of ordinals...

Hugh M. Hilden, Generators for two groups related to the braid group ...

Tom (Roy Thomas Jr.) Jacob, Some matrix transformations on analytic sequence

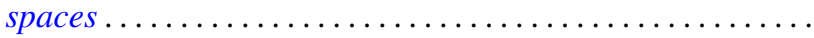

Elyahu Katz, Free products in the category of $k_{w}$-groups . .....

Tsang Hai Kuo, On conjugate Banach spaces with the Radon-Nikodým property...

Norman Eugene Liden, $K$-spaces, their antispaces and related mappings ...

Clinton M. Petty, Radon partitions in real linear spaces ........

Alan Saleski, A conditional entropy for the space of pseudo-Menger maps ....

Michael Singer, Elementary solutions of differential equations .

Eugene Spiegel and Allan Trojan, On semi-simple group algebras. I. . .

Charles Madison Stanton, Bounded analytic functions on a class of open Riemann

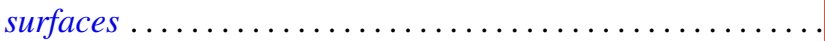

Sherman K. Stein, Transversals of Latin squares and their generalizations ....

Ivan Ernest Stux, Distribution of squarefree integers in non-linear sequences . . .

Lowell G. Sweet, On homogeneous algebras ................

Lowell G. Sweet, On doubly homogeneous algebras .......... .

Florian Vasilescu, The closed range modulus of operators ......

Arthur Anthony Yanushka, A characterization of the symplectic groups $\operatorname{PSp}(2 m, q)$

as rank 3 permutation groups... 